\title{
Promouvoir les services de vasectomie au Burundi
}

\author{
Brian Perry \\ Catherine Packer \\ Dawn S. Chin-Quee \\ Trinity Zan \\ Dominick Shattuck
}

Follow this and additional works at: https://knowledgecommons.popcouncil.org/departments_sbsr-rh How does access to this work benefit you? Let us know!

\section{Recommended Citation}

Perry, Brian, Catherine Packer, Dawn S. Chin-Quee, Trinity Zan, and Dominick Shattuck. 2016. "Promouvoir les services de vasectomie au Burundi." Durham, NC: FHI 360 and Washington, DC: The Population Council, The Evidence Project. 


\section{PROMOUVOIR LES SERVICES \\ DE VASECTOMIE AU BURUNDI}

Le mouvement de la Planification Familiale 2020 (FP2020) a commencé en 2012, lorsque plus de 150 dirigeants politiques, au Sommet de Londres sur la Planification Familiale (London Summit on Family Planning) se sont engagés à fournir l'accès aux contraceptifs d'ici 2020 à 120 million de femmes des pays les plus pauvres du monde. FP2020 vise à soutenir les droits reproductifs des femmes et des jeunes filles et leur donner les moyens d'achever leurs objectifs en matière de reproduction. Cela se fait, en partie, en s'assurant que les clientes de la PF à travers le monde reçoivent le choix éclairé d'un large éventail de méthodes contraceptives de haute qualité. Au cours de cette réunion, le gouvernement du Burundi s'est engagé à repositionner la PF comme une priorité nationale et à augmenter le taux de prévalence contraceptive parmi les femmes mariées à $50 \%$ d'ici 2020. ${ }^{1}$ De plus, le gouvernement s'est engagé à élargir l'accès aux services de PF, en augmentant les partenariats publicprivé, en formant des agents de santé sur la prestation de méthodes permanentes, réversibles à longue durée, et en sensibilisant sur l'importance de la PF. En 2014, le gouvernement du Burundi a appuyé le développement des capacités de 27 médecins et 27 infirmières dans les services opératoires pour la vasectomie ainsi que 14 médecins et 14 infirmières pour la ligature des trompes. ${ }^{2}$ L'extension de combinaison des méthodes grâce à renforcer la capacité, encourager la demande pour la vasectomie, et créer les services PF «pour les hommes» augmente la prévalence contraceptive moderne ${ }^{3}$ et aider le Burundi à atteindre ses objectifs en matière de PF.

Cependant, la gamme actuelle de méthodes de PF accessibles, les messages de changement de comportement et la communication interpersonnelle renforcent l'idée que les services de PF sont spécifiquement pour les femmes. Les idées fausses au sujet de la vasectomie, limitent le soutien financier, les politiques et le soutien communautaire, pour la méthode. En conséquence, les hommes sont insuffisamment engagés dans les services de santé de la reproduction et les femmes portent la responsabilité principale d'utiliser la contraception.

La vérité est que les services de vasectomie apportent aux couples cherchant à limiter la taille de leur famille, une alternative de PF très efficace, de nature permanente, et axée sur l'homme, sans effets secondaires.

Une plus grande intégration de la promotion et des services de vasectomie est une première étape dans l'expansion du paradigme de la santé de la reproduction pour inclure les hommes et pas seulement en tant que partenaires par défaut des clientes, mais en tant que bénéficiaire égal des programmes de santé de la reproduction.
La vasectomie sans bistouri:

- $\quad$ Est SANS DOULEUR, RAPIDE, et EXTRÊMEMENT EFFICACE

- Exige PEU DE RÉCUPÉRATION,

- Apporte DES AVANTAGES ÉCONOMIQUES ET SANITAIRES à la famille,

- N'A PAS D'EFFETS SECONDAIRES, et

- N'AFFECTE EN RIEN LA FONCTION SEXUELLE OU LA JOUISSANCE.

Une plus grande éducation et le soutien à la vasectomie au niveau national traiterait le déséquilibre entre les sexes dans la disponibilité et l'utilisation des contraceptifs. Contrairement à une nouvelle méthode concentrée sur la femme, l'intégration significative de la vasectomie dans une combinaison de méthodes élargit le paradigme de la santé de la reproduction pour inclure les hommes en tant qu'utilisateurs des services de la PF et promouvoir la fertilité des couples comme une responsabilité partagée. Cette approche plus holistique de la santé de la reproduction soutient un choix éclairé d'un large éventail de méthodes contraceptives de haute qualité.

La vasectomie est la méthode permanente la plus économique et la plus sûre pour répondre aux besoins des hommes et des femmes qui ne souhaitent plus avoir d'enfants. Vu l'engagement du Burundi à prioriser la PF et élargir l'accès et l'utilisation de méthodes modernes, maintenant, est le moment idéal d'investir dans les services de vasectomie. En favorisant et en soutenant l'utilisation volontaire de la vasectomie dans le cadre global de la combinaison des méthodes, le Burundi peut répondre à ses engagements nationaux et internationaux en matière de PF et contribuer au développement d'une société plus équitable en matière de genre, où les hommes jouent un rôle proactif et de soutien dans la santé de leurs familles.

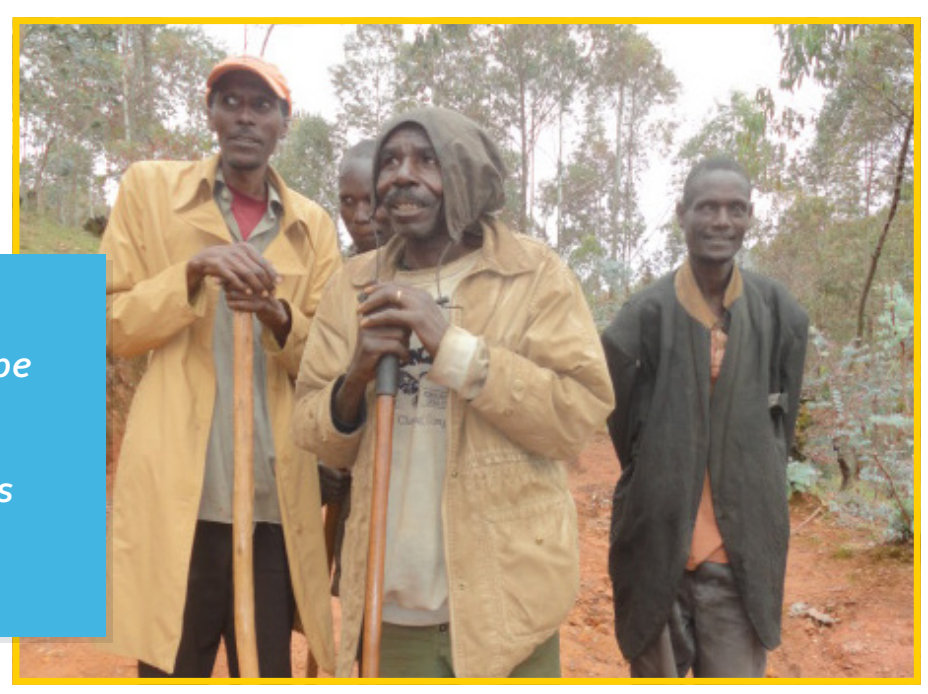




\section{LA VASECTOMIE AIDE LES PARTICULIERS ET LES COUPLES À ACHEVER LE NOMBRE D’ENFANTS DÉSIRÉ}

Au Burundi, $42 \%$ des hommes mariés et 34\% des femmes mariées en âge de procréer ont signalé un désir de limiter les futures naissances.4 Les hommes et les femmes plus âgés étaient beaucoup plus susceptibles de vouloir limiter les naissances (97\% de ces hommes et $91 \%$ de ces femmes étaient âgés de 30 ans au maximum) même si un nombre important de personnes plus jeunes sont aussi intéressées à limiter la taille de la famille.

Estimation de la taille du marché pour les méthodes permanentes, au Burundi, 20204,5

\begin{tabular}{|l|r|l|r}
\hline Population totale des femmes & $6,338,728$ & $6,293,246$ & Population totale des hommes \\
\hline Mariées (âgées de 15 à 49 ans) & $\mathbf{1 , 1 9 6 , 0 7 2}$ & $\mathbf{1 , 4 2 6 , 4 7 3}$ & Mariés (âgés de 15 à 59 ans) \\
\hline Désir de limiter les futures naissances* & $\mathbf{4 1 3 , 4 6 2}$ & $\mathbf{5 5 1 , 9 1 4}$ & Désir de limiter les futures naissances*
\end{tabular}

* Exclut les hommes et les femmes qui déclarent stérilisation antérieure ou infertilité

\section{LA VASECTOMIE ÉLARGIT LE CHOIX DES MÉTHODES EFFICACES PERMANENTES DE CONTRACEPTION}

Au Burundi, plus de 94\% des femmes mariées qui ont déclaré un désir de limiter les futures naissances utilisaient soit des méthodes contraceptives à court terme ou aucune méthode.4 Les injectables, qui ont un taux élevé d'abandon en raison d'effets secondaires, représentent $41 \%$ des contraceptifs utilisés par cette population. La vasectomie offre une durée de vie de la protection contraceptive sans soucis des effets secondaires.
Utilisation des contraceptifs chez les femmes âgées de 15 à 49 ans qui veulent limiter les futures naissances au Burundi ${ }^{4}$

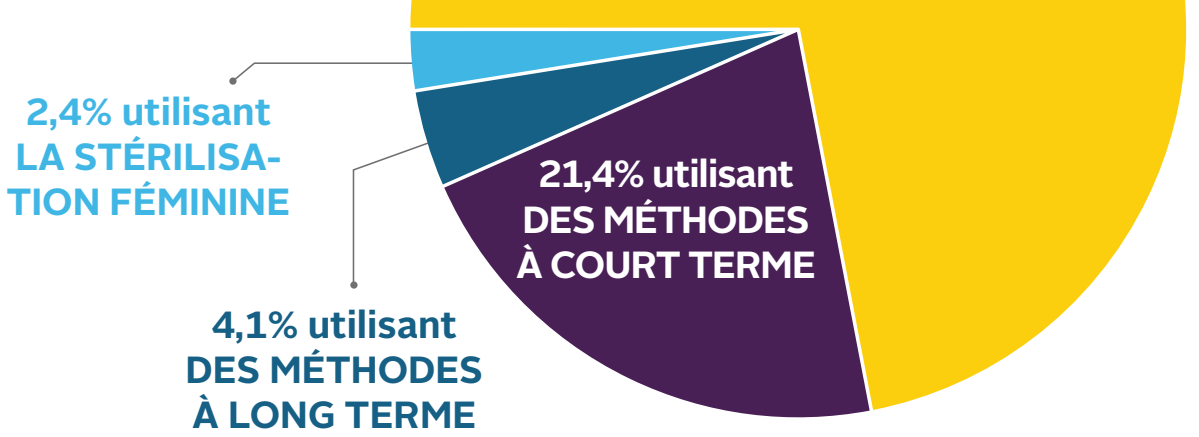

Les besoins non satisfaits de limitation: les couples qui veulent limiter les futures naissances et utilisent actuellement une méthode à court terme ou pas de méthode de contraception sont un important marché potentiel pour les services de vasectomie.

\section{2,1\% n'utilisant AUCUNE MÉTHODE}




\section{L’UTILISATION PLUS ÉLEVÉE DE VASECTOMIE RÉDUIT} LES TAUX DE GROSSESSES NON DÉSIRÉES ET LES COÛTS GLOBAUX DE SERVICES DE SANTÉ REPRODUCTIVE

Si le Burundi devait atteindre son objectif d'augmenter la prévalence contraceptive à 50\% parmi les femmes mariées (âgées de 15 à 49 ans) d'ici 2020, il y aurait environ un total de 600.000 utilisateurs de méthodes contraceptives dans ce groupe démographique. Si un faible pourcentage de besoins non satisfaits pour la limitation des couples mariés au Burundi a été satisfait grâce à une meilleure absorption de la vasectomie, cela aurait un impact direct et durable sur la santé publique et réduirait les coûts globaux des produits et des services de PF.

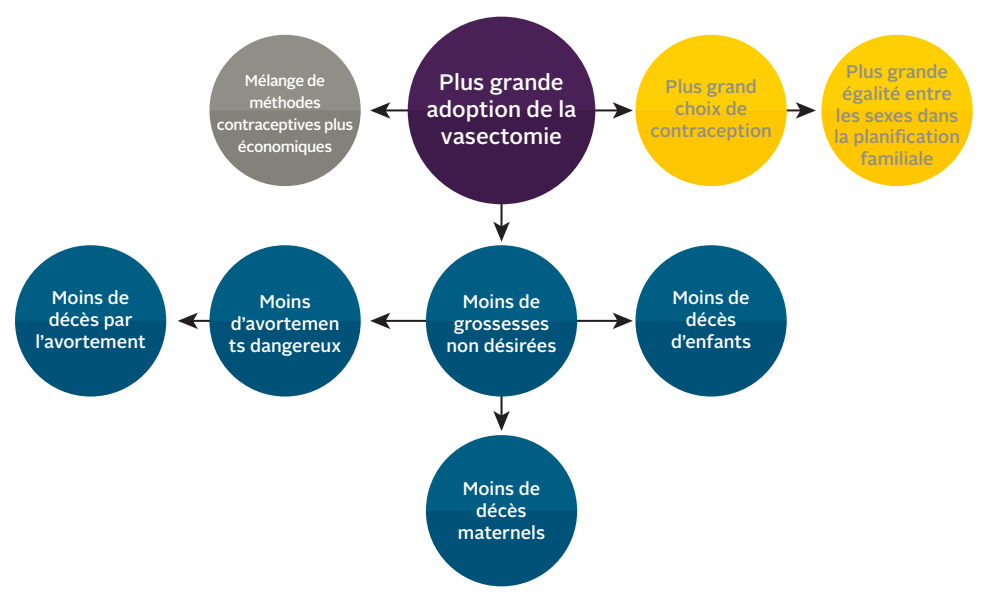

\section{LA VASECTOMIE EST PARMI LES MÉTHODES DE PLANIFICATION FAMILIALE LES PLUS ÉCONOMIQUES}

La vasectomie coûte moins ${ }^{a}$, que toutes autres formes de contraceptifs actuellement fournis par années de protection pour un couple, à l'exception des DIU au cuivre. ${ }^{6,7}$ Au Burundi, un investissement accru dans la vasectomie entrainerait plus d'économies par années de protection pour un couple en coûts de produits et service de la méthode de mélange contraceptive de la nation.

Coût de fourniture de contraceptifs par années de protection pour un couple en Haïti, 2015

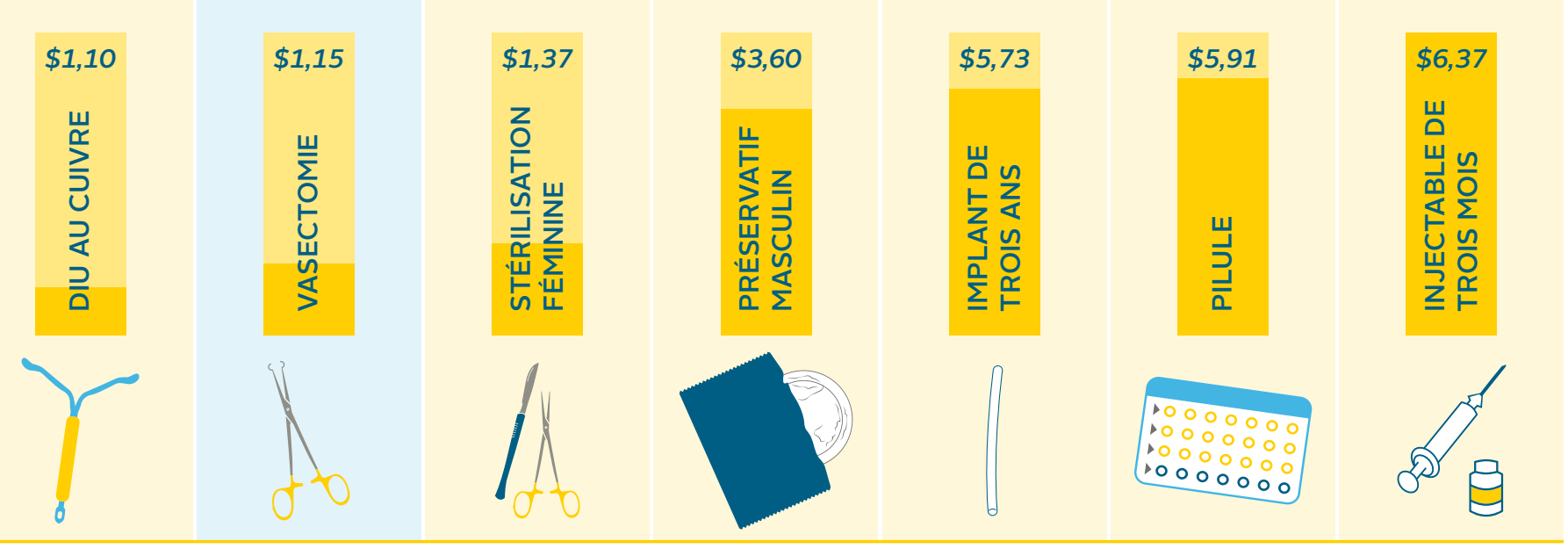

Les coûts par années de protection pour un couple ne reflètent que la main-d'oeuvre directe et le coût des matières premières et des fournitures consommables et ne prend pas en compte d'autres coûts potentiels associés à l'introduction de la vasectomie sur le marché, y compris la création de la demande, les formations de prestataire supplémentaires ou l'expansion de la couverture des services.

\section{LA VASECTOMIE PEUT CONTRIBUER À UNE COMBINAISON DE MÉTHODES MOINS COÛTEUSES ET PLUS EFFICACES}

Par exemple, si d'ici 2020, seulement 5\% des femmes mariées (âgées de 30 à 49 ans) qui souhaitent limiter les naissances passaient de l'utilisation de méthodes à courtterme comptant sur la vasectomie de leur partenaire pour achever leurs objectifs de fécondité, cela permettrait d'améliorer le rapport coût-efficacité du mélange de méthode pour ce groupe démographique par plus de 14\%.

Cette augmentation marginale de l'utilisation de la vasectomie pour limiter les naissances - égale à 15.390 des vasectomies réalisées au cours des 5 prochaines années permettrait d'améliorer l'impact du mélange de méthode sur la santé publique par plus de $18 \%$.

\section{CUMULATIF COUT-EFFICACITE ET IMPACTS SUR LA SANTE PUBLIQUE $(2016-2020)^{\mathrm{b}}$}

\begin{tabular}{|r|c|c|}
\hline & $\begin{array}{c}\text { Combinaison } \\
\text { de méthodes } \\
\text { actuelle* }\end{array}$ & $\begin{array}{c}\text { Combinaison de } \\
\text { méthodes* dont 5\% de } \\
\text { vasectomie prévalence } \\
\text { contraceptive }\end{array}$ \\
\hline $\begin{array}{r}\text { Coût par années de } \\
\text { protection pour un couple }\end{array}$ & $\$ 5,17$ & $\$ 3,99$ \\
\hline $\begin{array}{r}\text { Coût total par années de } \\
\text { protection pour un couple }\end{array}$ & 493.508 & 642.547 \\
\hline Grossesses non désirées évitées & 142.130 & 185.054 \\
\hline Décès maternels évités & 711 & 925 \\
\hline Décès infantiles évités & 8.386 & 10.918 \\
\hline Avortements dangereux évités & 42.298 & 55.072 \\
\hline Cas de décès à l'avortement évités & 220 & 286 \\
\hline
\end{tabular}

* Parmi les femmes mariées (30 à 49 ans) qui souhaitent limiter les naissances 


\section{CRÉER UN ENVIRONNEMENT FAVORABLE :}

- Mettre en place des politiques de soutien et un appui politique pour l'inclusion des hommes et élaborer un plan de mise en oeuvre budgétisé pour la PF qui comprend l'expansion des services de vasectomie et exige des activités de création.

- Combler l'écart entre les engagements de PF et le soutien financier disponible, les donateurs et le gouvernement devraient investir dans la combinaison de méthodes la plus rentable pour répondre aux besoins changeant des hommes, des femmes, et des couples sur la durée de leur vie reproductive.

- Adresser les normes liées au genre qui peuvent avoir un impact négatif sur la prise de décision en PF, par l'autonomisation des femmes et des couples pour parler ouvertement de leurs intentions en matière de reproduction et considérer la vasectomie comme une façon désirable d'assurer la santé et le bien-être de la famille.

- Capitaliser sur l'engagement du gouvernement à développer l'éducation à la sexualité pour les jeunes en incluant des informations sur les méthodes permanentes pour commencer la sensibilisation précoce sur la vasectomie comme une forme viable de limitation de naissances à l'avenir.

- Créer des services de santé de la reproduction "pour les hommes" en formant des agents de santé du sexe masculin pour conseiller les hommes au sujet de leurs options de PF.

\section{ACCROÎTRE L'OFFRE DE SERVICES DE VASECTOMIE:}

- Renforcer des capacités dans le secteur public aux endroits avec des installations d'enseignement médical et les services du secteur privé disponibles, et assez de demande des services pour soutenir le développement des compétences.

- Adresser les biais et les attitudes négatives potentielles des prestataires sur la prestation de services de vasectomie par la formation et les témoignages, et assurer que les prestataires reçoivent une compensation adéquate pour les services.

- Développer l'accès aux services de vasectomie et la disponibilité de prestataires formés en vasectomie grâce à la sensibilisation mobile et le transfert de tâches. Envisager de former des infirmiers pour procéder à la vasectomie sans bistouri avec l'utilisation de l'interposition aponévrotique et la cautérisation thermique.

- Investir dans le développement durable de compétences en vasectomie en formant des prestataires formateurs certifiés dans le secteur public comme formateurs de vasectomie.

\section{ENCOURAGER LA DEMANDE POUR LA VASECTOMIE:}

- Capitaliser sur l'engagement existant pour générer la demande de la vasectomie à travers différents canaux de communication, en particulier la radio, pour diffuser des informations exactes sur la vasectomie, particulièrement aux sujets de la sécurité de la méthode et son effet sur la virilité et la force physique.

- Engager et soutenir les "champions" de la vasectomie parmi les leaders religieux, politiques et communautaires, les prestataires de la santé, les agents de santé communautaires, et les clients satisfaits de la vasectomie.

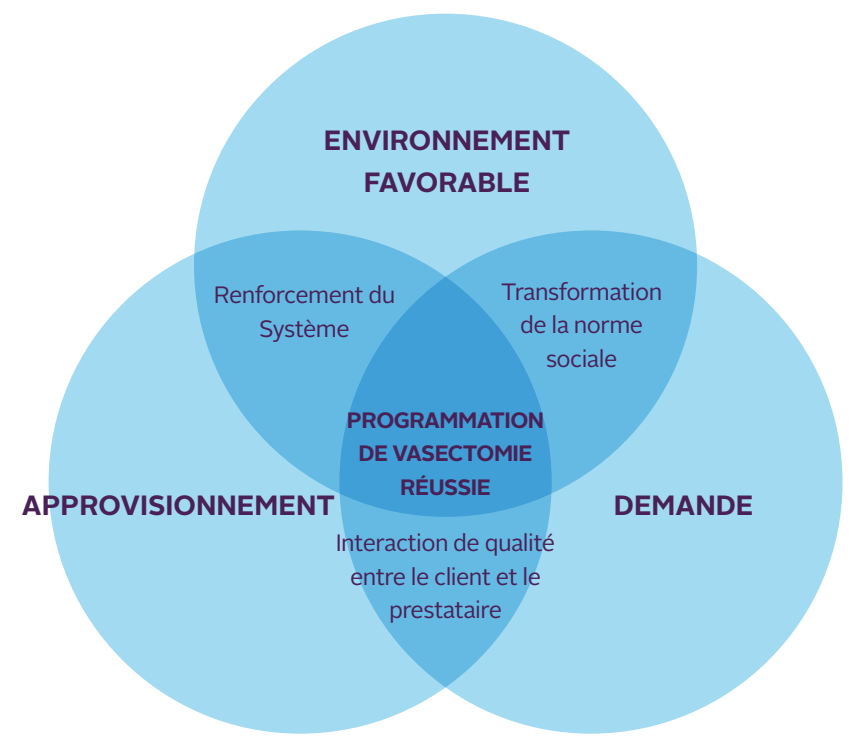

Adapté du Supply-Enabling Environment-Demand (SEED) ProgrammingModel ${ }^{\mathrm{TM}} \mathrm{d}^{\prime}$ EngenderHealth

Burundi FP2020 Commitments, Progress \& Transparency. 2014. Disponible à : http://www.familyplanning2020.org/entities/100

FP2020. Archived Commitments for Scale-up Progress Report: Burundi Official Update. 2015. Disponible à: http://www.familyplanning2020.org/topics/264/commitments Ross J and Stover J. Use of modern contraception increases when more methods become available: analysis of evidence from 1982-2009. Glob Health Sci Pract. 2013;1(2):203-212. Institut de Statistiques et d'Études Économiques du Burundi, Ministère des Finances et de la Planification du Développement Économique, Institut National de Santé Publique, Ministère de la Santé Publique et de la Lutte contre le Sida, and ICF International. 2012. Enquête Démographique et de Santé Burundi 2010. BUIR61FL.SAV and BUMR61FL.SAV. Bujumbura, Burundi and Calverton, Maryland: Institut de Statistiques et d'Études Économiques and ICF International [Producteurs]. ICF International [Distributeur], 2012.

uS Census Bureau. International Database. Dernière mise à jour juillet 2015. Disponible à http://www.census.gov/population/international/data/idb/informationGateway.php.

5. Tumlinson K, Steiner MJ, Rademacher KH, Olawo A, Solomon M, and Bratt J. 2011. The promise of affordable implants: is cost recovery possible in Kenya. Contraception, 83:88-93. Rademacher KH, Solomon M, Pascual C, Njunguru J, Brett T, and Steiner MJ. 2016. Expanding access to a new, more affordable levonorgestrel intrauterine system in Kenya: Perspectives from Key Opinion Leaders. Global Health: Science and Practice. In press.

8 Perry B, Packer C, Chin Quee D, Zan T, Dulli L, and Shattuck D. Recent experience and lessons learned in vasectomy programming in low-resource settings: a document review. Durham, NC: FHI 360 et Washington, DC: Population Council, the Evidence Project. 2016. 9 Packer C, Perry B, Chin-Quee D, Zan T, and Shattuck D. How to create successful vasectomy programs. Durham, NC: FHI 360 and Washington, DC: The Population Council, the Evidence Project. 2016.

Packer C, Perry B, Chin-Quee D, Zan T, and Shattuck D. How to create successful vasectomy programs. Durham, NC: FHI 360 and Washington, DC: The Population Council, the Evidence Project. 2016 


\section{LE PROJET EVIDENCE}

POPULATION COUNCIL

4301 Connecticut Avenue, NW, Suite 280

Washington, DC 20008 USA

Tel +1202 2379400

Evidenceproject.popcouncil.org

Ce travail est rendu possible grâce au soutien généreux du peuple américain à travers

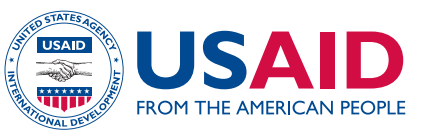

l'Agence Américaine pour le Développement International (USAID) aux termes d'un accord de coopération no. AID-OAA-A-13-00087 du Projet Evidence. Les résultats et les conclusions sont la seule responsabilité des auteurs et ne reflètent pas nécessairement les vues de I'USAID ou du gouvernement des États-Unis.

Le Projet Evidence vise à élargir l'accès aux services de haute qualité de planification

\section{Evidence} familiale/santé reproductive dans le monde entier grâce à la science de la mise en oeuvre, y compris la génération stratégique, la traduction, et l'utilisation de preuves nouvelles et existant. Le projet est dirigé par Population Council en partenariat avec le Réseau INDEPTH, I'International Planned Parenthood Federation, PATH, et le Population Reference Bureau.

\section{fhi 360}

FHI 360 est une organisation de développement humain sans but lucratif vouée à l'amélioration de la vie de façon durable en avançant des solutions intégrées, dirigées localement. Notre personnel comprend des experts en santé, en éducation, nutrition, environnement, développement économique, la société civile, le sexe, les jeunes, la recherche et la technologie - créant un mélange unique de capacités pour relever les défis de développement interdépendants d'aujourd'hui. FHI 360 sert plus de 60 pays, les 50 états américains et tous les territoires américains.

Pour plus d'informations sur les recommandations programmatiques sur la vasectomie, veuillez contacter:

\section{FHI 360}

Mailing address:

359 Blackwell Street,

Suite 200, Durham, NC 27701

Téléphone: +1 9195447040

Fax: +1 9195447261

Publié en Mars 2016

Citation Suggéré:

Perry B, Packer C, Chin-Quee D, Zan T, and Shattuck D. Promoting Vasectomy Services in Kenya. Durham, NC: FHI 360 and Washington, DC: The Population Council, the Evidence Project. 2016.

Copyright (c) 2016 FHI 360. Tous droits réservés. 


\section{PHOTO CITATIONS}

\section{Photo 1 - ID: 207-12}

Des ouvriers travaillent au Burundi pour élargir un chemin de terre de sorte que les camions puissant ramasser les arbres d'eucalyptus fraichement coupés des zones dénudées.

\section{(c) 2010 Jean Sack,}

Avec l'autorisation de Photoshare

\section{Photo 2 - ID: 2005-230}

Un homme âgé dans la province de Muramvya au Burundi recueille les feuilles de thé dans les plantations Tesa. Thé est la perle verte du Burundi.

(c) 2004 Isabelle Walhin, Avec l'autorisation de Photoshare 